\title{
"Na Sombra da Doença": A Perspetiva de Irmãos de Crianças em Cuidados Paliativos
}

\author{
"In the Shadow of the Disease": The Perspective of Siblings of Children in Palliative \\ Care
}

\author{
Alexandra Jóni Nogueira ${ }^{1}$ e Maria Teresa Ribeiro ${ }^{2}$
}

\begin{abstract}
Resumo
O presente estudo, de natureza descritivo-exploratória, pretendeu contribuir para a compreensão da experiência psicológica da doença crónica complexa na fratria em idade escolar. A amostra foi constituída por sete famílias de crianças em cuidados paliativos, tendo seis mães e uma avó preenchido um questionário sócio demográfico. Foram entrevistados nove irmãos saudáveis, com idades entre os 6 e os 9 anos, através de uma história infantil ilustrada e da elaboração de uma narrativa baseada no seu desenho sobre a fratria. Os resultados da análise qualitativa evidenciam oito categorias principais: compreensão da doença, representação do irmão doente, impacto emocional, ocupação de tempos livres e responsabilidades, perspetivas sobre o futuro, relação fraterna, dinâmicas familiares e fratria imaginária. A reduzida dimensão da amostra e a diversidade de doenças constituem limitações deste estudo. Retiram-se implicações para a intervenção psicológica na fratria, sugerindo-se investigações futuras que explorem a rivalidade fraterna e outras faixas etárias.
\end{abstract}

Palavras-chave: cuidados paliativos pediátricos, doença, irmãos, família, experiência psicológica

\begin{abstract}
The present study, of descriptive-exploratory nature, aimed to contribute to the understanding of the psychological experience of complex chronic disease in school-age siblings. The sample consisted of seven families of children in palliative care, composed by six mothers and one grandmother which filled out a socio-demographic questionnaire. This was followed by the participation of nine healthy siblings, aged between 6 and 9 years, through an illustrated children's story, and the elaboration of a narrative based on their drawing about siblings. The results from qualitative analysis evidenced eight main categories: understanding of the disease, sick sibling representation, emotional impact, leisure time occupation and responsibilities, future perspectives, fraternal relationship, family dynamics and imaginary siblings. The reduced sample dimension and the diversity of diseases are considered some of the study's limitations. For the psychological intervention, implications are drawn suggesting further research exploring the siblings' rivalry and other age groups.
\end{abstract}

Keywords: pediatric palliative care, illness, siblings, family, psychological experience

\footnotetext{
${ }^{1}$ Mestre em Psicologia Clínica e da Saúde e Pós-Graduada em Cuidados Paliativos Pediátricos. Psicóloga clínica no Serviço à Comunidade da Faculdade de Psicologia da Universidade de Lisboa, Lisboa, Portugal. (Ano Profissional Júnior da Ordem dos Psicólogos Portugueses). Tel.: +351910897654. E-mail: alexandra.jnog@ gmail.com

${ }^{2}$ Doutorada em Psicologia. Professora Associada, Faculdade de Psicologia da Universidade de Lisboa. Alameda da Universidade, 1649-013 Lisboa, Portugal. Tel.: +351968091599. E-mail: mteresaribeiro@psicologia.ulisboa.pt

Revista Iberoamericana de Diagnóstico y Evaluación - e Avaliação Psicológica. RIDEP · No54 · Vol.1 · 19-33 · 2020

ISSN: 1135-3848 print /2183-6051online
} 


\section{Introdução}

Os cuidados paliativos pediátricos (CPP), reconhecidos pela Organização Mundial de Saúde desde 1988, constituem um direito humano básico que deve ser reconhecido a qualquer criança ou adolescente com uma doença crónica complexa ou grave (Goldman, Hain, \& Liben, 2012). A mesma deverá afetar um órgão em específico ou diferentes sistemas, de forma suficientemente grave, durando há pelo menos 12 meses e requerendo cuidados especializados (Feudtner, DiGiuseppe, \& Neff, 2003). Por este motivo, a doença tem um impacto significativo na qualidade de vida e/ou esperança de vida da criança ou jovem e da sua família. Os CPP devem ser prestados durante todo o ciclo de vida, desde o processo de diagnóstico até ao período pós-morte, intervindo a nível físico, emocional, social e espiritual, com o objetivo de melhorar a qualidade de vida não só da criança doente, mas também de todo o sistema familiar (World Health Organization [WHO], 1998).

A entrada da doença no ciclo vital familiar conduz, inevitavelmente, a alterações na experiência psicológica e conduta de cada membro perante esta adversidade, bem como na família como um todo (Carter \& McGoldrick, 2005), produzindo um impacto significativo em toda a estrutura e dinâmica familiares. Este acontecimento de vida, stressante e perturbador, irá influenciar o processo de desenvolvimento e adaptação de cada elemento e da família, sendo simultaneamente determinado e determinando pelo meio em que vive (Barros, 2003).

\section{A experiência psicológica dos cuidadores familiares}

Nas últimas décadas, os avanços científicos ao nível dos cuidados e tecnologia médica têm permitido prolongar a vida das crianças com condições de saúde clinicamente complexas. As instituições de saúde eram responsáveis por prestar os cuidados necessários à maioria destas crianças. Atualmente, a prestação de cuidados é assegurada pela família, no domicílio (Mesman, Kuo, Carroll, \& Ward, 2013). Os familiares cuidadores da criança - maioritariamente os progenitores - são uma peça fundamental, ao assumirem a responsabilidade primária da mesma, ajudando-a a realizar todas as tarefas que esta não consegue desempenhar autonomamente.

Nesta sequência, as investigações têm-se centrado sobretudo na compreensão da adaptação psicológica no subsistema parental (e.g., Navarausckas, Sampaio, Urbini, \& Costa, 2010). A literatura tem reforçado o impacto desta experiência ao nível do seu bem-estar e qualidade de vida. Segundo Nogueira e Francisco (2017), esta é afetada de forma maioritariamente negativa em todas as suas dimensões, verificando-se: cansaço frequente e stress na realização de atividades (dimensão física); preocupações constantes relativas à prestação de cuidados e antecipação do futuro, bem como culpabilização pela falta de atenção aos filhos saudáveis, em contraste com a satisfação perante as melhorias e conquistas dos filhos que estão doentes (dimensão psicológica); privação de oportunidades de lazer e socialização e desconforto perante os estereótipos e juízos de valor da sociedade (dimensão social); e dificuldades de acesso a serviços de saúde, pela sua escassez ou encargo financeiro (dimensão social). A maioria destas famílias encontra-se numa situação socioeconómica fragilizada, por um lado devido aos encargos com a assistência terapêutica e os materiais de apoio que a criança ou jovem necessita, e por outro lado porque apenas um cuidador assume a responsabilidade de sustento de toda a família (Mesman et al., 2013).

Para além disso, com frequência, os pais sentem desesperança em relação ao futuro, superprotegem o filho doente e manifestam medo da morte (Azeredo, Amado, Silva, Marques, \& Mendes, 2004). Sentem-se impotentes por não conseguirem ajudar o filho doente a sentir-se melhor e têm pensamentos intrusivos (Rodriguez et al., 2012). Estes cuidadores familiares apresentam também sintomatologia associada a stress pós-traumático, a qual tende a aumentar nos primeiros 18 meses após o diagnóstico, e a diminuir a partir dos 5 anos de experiência diária com o mesmo (Jurbergs, Long, Ticona, \& Phipps, 2009).

\section{A experiência psicológica na fratria}

$\mathrm{O}$ processo de doença crónica complexa é vivenciado de forma diferente por todos os elementos do sistema familiar, pelo que a presença de outros filhos na família implica a 
necessidade de conhecer a forma como experienciam esta condição na fratria. A adaptação é um processo contínuo e dinâmico, com fases de maior equilíbrio e aceitação, e outras de maior ansiedade, revolta ou depressão, seguindo padrões individuais dificilmente generalizáveis (Barros, 2003). As características das doenças crónicas complexas em crianças e jovens, a sua gravidade e trajetória de evolução, e também a necessidade de períodos de hospitalização, a par com os diversos tratamentos disponíveis, são determinantes na capacidade adaptativa de cada um dos familiares (Pelchat et al., 2001).

Desde os anos 70 que se tem observado um acréscimo do interesse na investigação do impacto da doença crónica nos irmãos saudáveis. A forma como os adultos gerem e se adaptam ao processo de doença afeta o entendimento da própria criança saudável e tem implicações na forma como esta perspetiva o adoecimento e as mudanças que daí decorram nas suas vidas (Cardoso, 2007). Assim, é expectável que a resposta emocional dos irmãos saudáveis acompanhe a resposta da sua família.

Os irmãos saudáveis têm de se adaptar às mudanças no funcionamento familiar, à fadiga física e emocional dos pais e ao seu próprio turbilhão emocional. Com frequência, têm de gerir as alterações ao nível da relação fraterna e, em alguns casos, do papel que passam a assumir, frequentemente com exigências de natureza parental (Brown, Coad, \& Franklin, 2017). Numa perspetiva sistémica, a presença de uma criança com uma doença crónica complexa na família pode perturbar a educação dos seus irmãos em três áreas principais: na interação e relação fraterna, na organização familiar e no papel assumido pelos irmãos saudáveis (Mendonça, 2000).

A literatura tem também reforçado que a experiência psicológica da doença na fratria tem implicações ao nível do bem-estar psicológico dos irmãos saudáveis, podendo ser percursora de perturbações emocionais internalizadas e externalizadas (Vermaes, van Susante, \& Bakel, 2012). Por também poder ser considerada um acontecimento de vida potencialmente traumático (Miceli \& Zornig, 2002), é fundamental conduzir estudos que explorem e visem uma compreensão mais aprofundada desta temática, atualmente em desenvolvimento.
É igualmente pertinente considerar as estratégias de coping dos irmãos saudáveis, as quais variam em função de fatores como a idade, capacidade de compreensão da situação de doença, experiências de vida, cultura e temperamento. Contudo, existem condutas que podem influenciar positivamente o seu ajustamento psicológico, destacando-se a promoção de uma comunicação aberta e honesta, a transmissão de informação sobre a doença, condição e tratamentos da criança doente, e também o desejo demonstrado pelos irmãos em estarem envolvidos ativamente no cuidado da criança doente (Björk, Wiebe, \& Hallström, 2005; Woodgate, 2006).

Todavia, e apesar das implicações negativas que a doença crónica complexa na fratria pode acarretar, a literatura destaca também o impacto positivo decorrente desta experiência psicológica. Segundo Barros (2003), esta pode constituir uma oportunidade para estimular o desenvolvimento eficaz de forças e competências. Destacam-se um maior nível de sensitividade, compreensão e literacia em saúde (Fleary \& Heffer, 2013), responsabilidade cívica, atitudes empáticas e compassivas, desejo de apreciar a vida e espiritualidade (Alderfer et al., 2010).

Numa perspetiva de promoção dos aspetos positivos resultantes desta experiência adversa no ciclo vital da família, e simultaneamente de prevenção do seu impacto negativo, é fundamental refletir-se sobre a intervenção psicológica junto dos irmãos saudáveis. $\mathrm{O}$ interesse nesta intervenção tem vindo a aumentar significativamente, sobretudo através do desenvolvimento e implementação de programas em grupo. Se, para os pais, os efeitos são evidentes na diminuição da sua angústia e no aumento da sua estabilidade psicológica e bemestar geral (Pai, Drotar, Zebracki, Moore, \& Youngstrom, 2006; Svavarsdottir \& Sigurdardottir, 2006), para os irmãos, o impacto reflete-se sobretudo ao nível da satisfação das suas necessidades de apoio emocional, social e informativo (Murray, 2002).

Prchal e Landolt (2009) realizaram uma revisão de literatura sobre as intervenções psicológicas com estes irmãos - individualmente, por acampamento e em grupo -, concluindo que todas as modalidades contribuem para uma 
diminuição da sintomatologia de ansiedade e depressão manifestadas, a par com um maior nível de conhecimento e compreensão da doença. Outros estudos destacam também um aumento da sua qualidade de vida (Besier, Hölling, Schlack, West, \& Goldbeck, 2010) e um maior envolvimento com os irmãos doentes (Hartling et al., 2014).

Esta conjugação de fatores sugere que os irmãos saudáveis constituem uma população de risco, reforçando a pertinência de estudar as suas vivências no âmbito da doença crónica complexa na fratria. Este estudo deve partir da sua própria perspetiva, evitando fontes secundárias, uma vez que os irmãos são quem melhor conhece a sua própria experiência psicológica. Para além disso, os irmãos em idade escolar, entre os 6 e os 12 anos, são capazes de expressar verbalmente os seus sentimentos e de narrar as suas experiências, validando assim o seu discurso em relação à doença e à fratria (Carr, 2006).

\section{Objetivos de investigação}

Fundamentando-se em tais considerações, o presente estudo surge com o intuito de responder à lacuna existente na literatura revista e de conhecer a experiência dos irmãos através da sua própria perspetiva. Nesse seguimento, a questão de investigação orientadora deste estudo, que reflete o seu objetivo geral, é: "Qual a experiência psicológica da doença crónica complexa na fratria em idade escolar?".

Como objetivos específicos, pretende-se explorar:

a) A sua compreensão da doença;

b) As suas representações do irmão doente;

c) O impacto emocional da doença;

d) A ocupação que faz dos seus tempos livres e as responsabilidades associadas;

e) As suas perspetivas sobre o futuro, nomeadamente em relação a si e à sua família;

f) As dinâmicas da relação fraterna.

Ao permitir explorar as especificidades da experiência psicológica dos irmãos saudáveis, esta investigação favorece a identificação das suas necessidades específicas, resultando no desenvolvimento de competências promotoras da sua resiliência e crescimento pós-traumático. Possibilita ainda um reforço do papel dos irmãos e da relação fraterna no processo de adaptação à doença crónica com necessidade de cuidados paliativos pediátricos. Por último, permite um apoio mais sólido e eficaz no ajustamento das práticas parentais, favorecendo assim uma visão holística e sistémica desta experiência.

\section{Método}

\section{Participantes}

Cuidadores principais. A amostra deste estudo é composta por um total de sete famílias de crianças com diagnóstico de uma doença crónica complexa, através da participação inicial dos seus cuidadores principais. Os mesmos têm idades compreendidas entre os 27 e os 57 anos, sendo a média de 38,29 $(D P=9,46)$. Todos são do sexo feminino e de nacionalidade portuguesa, sendo que seis são mães e uma é avó. Quanto ao estado civil, seis $(85,7 \%)$ são casadas e uma $(14,3 \%)$ é viúva. Relativamente às habilitações literárias, metade tem entre o $4 .^{\circ}$ e o $6 .^{\circ}$ ano de escolaridade e metade completou o ensino superior. Quanto à sua situação laboral, duas $(28,6 \%)$ estão desempregadas, três $(42,9 \%)$ encontram-se a dar assistência permanente ao filho com doença crónica e duas $(28,6 \%)$ estão empregadas.

Crianças com doença crónica complexa. Todas as crianças e jovens são do sexo masculino e as suas idades variam entre 4 e 13 anos, sendo a média de 6,43 anos $(D P=3,41)$. Atendendo aos grupos de diagnóstico em CPP (Association for Children with Life-Threatening or Terminal Conditions and their Families and the Royal College of Paediatrics and Child Health [ACT \& RCPCH], 2009), duas inserem-se no Grupo I (e.g., neurofibromatose I com glioma das vias óticas), um no Grupo II (e.g., fibrose quística), um no Grupo III (e.g., bulbomielite idiopática com insuficiência respiratória) e três no Grupo IV (e.g., paralisia cerebral profunda). De entre estas crianças, quatro $(57,1 \%)$ receberam o diagnóstico da doença até há 5 anos e todas foram sujeitas a hospitalizações, numa média de 6,57 vezes $(D P=7,27)$. Para cinco $(71,4 \%)$ destas crianças, a sua última hospitalização decorreu até há um ano atrás. Todas recebem atualmente apoios institucionais, destacando-se o ambulatório $(28,6 \%)$, o apoio material $(14,3 \%)$, os cuidados de 
enfermagem e fisioterapia $(14,3 \%)$ e o transporte $(14,3 \%)$.

Irmãos saudáveis. O agregado familiar é composto por entre três e seis elementos, com uma média de 4,57 ( $D P=0,98)$, sendo que em cinco $(71,4 \%)$ famílias estes são a mãe, o pai e $\mathrm{o}(\mathrm{s})$ irmã(o) saudável(eis). Quanto a estes últimos, em quatro $(57,1 \%)$ casos, as crianças doentes têm apenas um irmão saudável, em dois $(28,6 \%)$ têm dois irmãos saudáveis e apenas em um $(14,3 \%)$ caso há três irmãos saudáveis. Dos irmãos saudáveis que participaram no presente estudo, num total de nove crianças, três $(33,3 \%)$ são do sexo feminino e seis $(66,7 \%)$ são do sexo masculino. Têm idades compreendidas entre os 6 e os 9 anos, com uma média de 7,44 anos ( $D P=$ 1,33). Todas estas crianças frequentam a escola, sendo que três estão no $1 .^{\circ}$ ano, uma no $2 .^{\circ}$ ano, duas no $3 .^{\circ}$ ano, duas no $4 .^{\circ}$ ano e uma no $5 .^{\circ}$ ano. Todas vivem com o irmão doente e todas têm conhecimento da sua doença.

\section{Instrumentos}

Questionário Sócio Demográfico. Pretende recolher informações que caracterizem a amostra do estudo, nomeadamente características sócio demográficas do cuidador principal (e.g., sexo, idade, situação civil, grau de parentesco), da criança doente (e.g., idade, hospitalizações, diagnóstico da doença), da família (e.g., agregado familiar, idades) e dos irmãos saudáveis (e.g., sexo, idade, grau de conhecimento da doença, coabitação).

Guião de entrevista semiestruturada à criança. $O$ presente guião foi construído sob a forma de uma história infantil ilustrada sobre o Joãozinho, uma personagem fictícia cujo irmão é uma criança com doença crónica complexa. A história aborda alguns domínios da sua vida, como as rotinas em casa, as dinâmicas escolares, a interação com pares e a vida familiar. Este guião foi elaborado a partir de uma revisão da literatura no âmbito do processo de adaptação à doença crónica na fratria e estrutura-se em cinco blocos temáticos: compreensão da doença; representação do irmão doente; impacto emocional; ocupação de tempos livres e responsabilidades; e perspetivas sobre o futuro. Assim, e sob a forma de um livro, é apresentada uma narrativa sobre o Joãozinho, sendo intercaladas algumas questões. Para cada domínio descrito, existe uma questão que o pretende explorar através da resposta da criança. $\mathrm{O}$ recurso a histórias incompletas permite uma compreensão mais aprofundada do universo emocional das crianças, sobretudo ao nível das suas expetativas e representações das suas relações familiares (Román, Hodges, Palacios, Moreno, \& Hillman, 2018).

Construção de uma narrativa a partir do desenho da fratria. Solicita-se à criança que elabore um desenho sobre si e o(s) seu(s) irmão(s), sendo-lhe posteriormente pedida a elaboração de uma narrativa sobre o mesmo, colocando-se para isso uma questão orientadora: "Podes contar-me a história do teu desenho?" (White, 2005). Em nenhum momento o desenho foi analisado, uma vez que o único objetivo é aceder à narrativa sobre a fratria.

\section{Procedimento}

Após a avaliação das condições éticas do estudo, por parte da Comissão Especializada de Deontologia do Conselho Científico da Faculdade de Psicologia da Universidade de Lisboa, foi disponibilizado o questionário sócio demográfico online. Este foi divulgado nas redes sociais, utilizando-se uma amostra de conveniência nãoprobabilística, constituída pelo método "bola de neve". Foi também feito um apelo a diversas Instituições de Saúde, tendo havido colaboração na divulgação por parte da Associação Nacional de Fibrose Quística, da Associação de Paralisia Cerebral de Lisboa e da Associação Pais-emRede.

Todos os participantes assinaram o consentimento informado, disponibilizado por escrito na plataforma, e responderam voluntariamente ao questionário, tendo fornecido um contacto pessoal para possibilitar dar continuidade à investigação. Como critérios de inclusão, todas as crianças participantes, com idade entre os 6 e os 12 anos, deviam ser irmãs saudáveis de crianças ou jovens diagnosticados com uma doença que exige cuidados paliativos abrangida por um dos quatro grupos identificados na literatura (ACT \& RCPCH, 2009) -, há pelo menos 12 meses. Deviam também ter conhecimento da existência da doença e viver com o seu irmão doente - desde o nascimento, no caso de irmãos mais velhos. 
Aquando da recolha de dados com os irmãos saudáveis, foi novamente assinado um consentimento informado pelo cuidador principal, através de um documento escrito. Foi também solicitada autorização para a realização de uma gravação de áudio da mesma, assegurando-se questões éticas. Era igualmente solicitado um consentimento oral por parte da criança, bem como transmitido os seus direitos, nomeadamente à desistência e à não-resposta. As atividades foram realizadas maioritariamente no domicílio das famílias.

Posteriormente, procedeu-se à transcrição das várias narrativas e à sua análise temática, num processo indutivo-dedutivo (Braun \& Clarke, 2006), recorrendo-se ao software NVIVO 10. Para a abordagem quantitativa, utilizada na caracterização sócio demográfica das famílias, recorreu-se ao software estatístico SPSS, versão 21.

\section{Resultados e Discussão}

A análise dos dados resultou num sistema de categorias, num total de 206 itens, sendo oito o número das categorias principais. Entre estas, salientam-se aquelas que pretendem responder aos objetivos específicos do presente estudo Compreensão da doença, Representação do irmão doente, Impacto emocional no irmão saudável, Ocupação de tempos livres e responsabilidades, Perspetivas sobre o futuro e Relação fraterna - e aquelas que emergiram adicionalmente durante a análise dos dados, devido ao carácter descritivoexploratório deste estudo - Dinâmicas familiares e Fratria imaginária. Seguidamente apresentar-se-á cada uma das categorias principais, elucidando-se sobre as suas subcategorias mais frequentes $\mathrm{e}$ discutindo os resultados à luz da literatura na área. Salienta-se que não existe um critério definido para a ordem de apresentação das subcategorias.

\section{Compreensão da doença}

A literatura tem reforçado que a compreensão da situação de doença do irmão e das suas implicações influencia a capacidade de adaptação das crianças saudáveis. Contudo, ainda existe bastante relutância por parte dos pais na transmissão de informações (Essen \& Enskär,
2003), resultando, com frequência, na falta de explicação sobre a necessidade de a criança doente receber mais atenção e cuidados especiais. Para Seligman (1983), esta representa uma das principais razões para a sintomatologia de ansiedade manifestada pelos irmãos saudáveis.

Conhecimento da doença. Embora todos os participantes identifiquem a existência de uma doença, apenas dois apresentam conhecimento sobre o seu diagnóstico, no sentido de identificarem do que se trata a doença. No último caso é, contudo, evidente que existe uma dificuldade em explicar, por palavras próprias, as características da mesma: "Já não me lembro qual era a doença. A doença do mano é... não me lembro do nome. 'Fu' qualquer coisa... é fibrose. Fibrose quística. Hum, não consigo explicar o que é... (J., irmão com 9 anos de criança com fibrose quística)". As outras sete crianças deste estudo manifestam desconhecimento sobre o diagnóstico, associado a informação bastante limitada e imprecisa: "[Se fosse o mano, dizia] que ele tinha uma doença no olho... um dói-dói no olho que, se ficar muito forte, ele ficava cego (I., irmã com 7 anos de criança com neurofibromatose I com glioma das vias óticas)". Estes resultados são corroborados por Rocha (2015) que concluiu, no seu estudo, que os irmãos apenas acedem à informação que os seus pais partilham consigo, maioritariamente sobre aspetos básicos da doença e observáveis pelos próprios. Todavia, é importante salientar que a transmissão de informação sobre a doença, condições e tratamentos tem sido identificada como uma estratégia de coping eficaz (Björk et al., 2005). Acrescenta-se ainda que apenas uma criança mencionou ter noção da irreversibilidade da doença.

Referência às complicações associadas à doença. A maioria dos participantes referiu as dificuldades digestivas e respiratórias, a tosse, a febre, a gripe, a constipação e os vómitos como manifestações de doença dos irmãos (Bernardes \& Pegoraro, 2015): “O irmão do Joãozinho está constipado. [Para curar] é preciso dar-lhe remédio (C., irmã com 6 anos de criança com paralisia cerebral profunda)".

Necessidades do irmão doente. Oito das crianças participantes fizeram referência às necessidades identificadas no irmão doente, dando 
destaque ao descanso: "Ele tem uma doença... e tem que tomar remédio. E tem que estar na cama (G., irmão com 8 anos de criança com trissomia 21 associada a cardiopatia congénita, dificuldades renais e respiratórias e hipotiroidismo)". Por sua vez, quatro destas crianças fizeram referência à medicação regular, mencionando ainda a sua deslocação por cadeira de rodas e a necessidade de manter brincadeiras controladas (Lobato \& Kao, 2002).

Prestação de cuidados pela família. Foi também feita referência à prestação de cuidados pela família (Mesman et al., 2013). Três dos irmãos saudáveis abordaram o cuidado por parte da sua família: "O T. [nome do irmão doente] estava na cama, porque estava doente. E a médica mandou-lhe estar na cama. E a minha mãe cumpriu as ordens e a médica deu-lhe xaropes para ele tomar e ele tomou e ficou melhor (P., irmão com 9 anos de criança com paralisia cerebral profunda)".

Recurso aos profissionais de saúde. A citação anterior apela também ao recurso da família aos profissionais de saúde. Em nenhum momento foi feita referência à sua própria procura de apoio junto destes profissionais. Segundo Patterson, Millar e Visser (2011), num estudo sobre as necessidades que os irmãos de criança com doença crónica não veem satisfeitas, os seus participantes destacaram a falta de acesso a serviços de apoio e ajuda profissional. McDonald, Patterson, White, Butow e Bell (2014) complementam esta informação com as suas conclusões de que estas necessidades não atendidas pelas instituições de saúde a que recorreram são mais intensas quando o tratamento do irmão doente se prolonga no tempo ou quando existem recaídas.

Por último, duas crianças manifestaram o desejo de se manterem informadas relativamente à doença do seu irmão, sendo que uma destas fez também referência à necessidade de hospitalizações: "Se calhar tinha ... pronto, estava doente. Tinha uns bichos dentro dele (aponta para o corpo todo). Dizia que queria perceber o que é que ele tinha... e as coisas que ele podia fazer, mas sempre... porque quando ele ia sempre para o hospital, estava sempre a doer (S., irmã com 6 anos de criança com bulbomielite idiopática com insuficiência respiratória com dependência total de ventilação)". Segundo a literatura, a necessidade de hospitalizações, e por isso a trajetória de evolução e as agudizações da doença, são determinantes importantes na capacidade de adaptação à doença (Pelchat et al., 2001). Para além disso, Woodgate (2006) reforça o desejo que os irmãos saudáveis manifestam em estarem atualizados em relação à condição do seu irmão e em envolverem-se ativamente no seu cuidado.

\section{Representação do irmão doente}

Para descreverem o irmão doente, a maioria das crianças fez referência ao seu nome, à idade e à aparência física. Duas crianças destacaram o sentido de normalidade que a condição de saúde do seu irmão doente poderia assumir e três destacaram o seu sentido de diferença: " $E$ um prazer apresentar o meu irmão. Para vocês verem, para saberem coisas sobre ele. E para saberem que ele é diferente de nós. Que é diferente de nós, mas em algumas coisas é igual (P., irmão com 9 anos de criança com paralisia cerebral profunda)".

Referência à doença. Aquando da apresentação do irmão doente aos seus pares, três crianças referiram a doença como uma característica: "[O Joãozinho] pode ter dito 'Ele é doente e não consegue andar. E chama-se... Chama-se André (G., irmão com 9 anos de criança com síndrome genético ATR-x)". Por outro lado, cinco das crianças abordaram a doença como sendo uma situação externa ao seu irmão: "Estava doente, com febre e estava a vomitar. Porque apanhou uma gripe (P., irmão com 9 anos de criança com paralisia cerebral profunda)". Em ambos os casos, existiu uma identificação de incapacidades resultantes da doença, sendo estas assumidas como características, como a falta de mobilidade e a ausência de comunicação verbal: "Olá! Este é o Q. [nome do irmão doente]. Não anda e não fala. Não se consegue mexer. Anda na cadeira de rodas e... está doente... está sempre doente (D., irmão com 6 anos de criança com paralisia cerebral profunda)". Seligman (1983) destaca a possibilidade de existir ansiedade dos irmãos saudáveis em relação às incapacidades com que se confrontam por parte da criança doente, constituindo esta uma das primeiras fontes de perturbação psicológica. 
Atribuição de características positivas. Quatro das crianças participantes descreveram o seu irmão doente recorrendo a características positivas, como ser "fixe", ser "fofinho" e ser "capaz". Apesar da literatura identificar a vergonha como uma emoção frequente dos irmãos saudáveis, relativa sobretudo à aparência física do seu irmão ou a características resultantes da sua doença (Gomes, Lima, Rodrigues, Lima, \& Collet, 2013), é de salientar que dois irmãos caracterizaram o seu irmão como sendo "bonito".

\section{Impacto emocional no irmão saudável}

Os irmãos saudáveis fazem referência às suas vivências emocionais, no âmbito do processo de adaptação à doença do irmão, num total de 66 vezes. De seguida apresentam-se as subcategorias mais frequentes.

Alegria. Cinco crianças destacam a diversão que sentem com a brincadeira na fratria, tanto com o irmão doente, como com os outros irmãos saudáveis, quando existem. Para além disso, algumas crianças referem sentir alegria pela convivência regular com o irmão doente - em casa e na escola - e quando identificam características positivas em si próprias. A alegria é ainda referida quando têm a oportunidade de falar sobre o irmão, quando este pode regressar a casa após um período de hospitalização, quando participam em atividades de lazer e quando percecionam que a família tem possibilidade de responder financeiramente às exigências da prestação de cuidados: "Pode sentir-se feliz porque a família não é pobre e consegue pagar os tratamentos dele (G., irmão com 9 anos de criança com síndrome genético ATR-x)". Opperman e Alant (2003) referem que irmãos que pertençam a família com um estatuto sócio económico elevado parecem apresentar uma melhor adaptação do que irmãos integrados em famílias com um estatuto sócio económico mais baixo.

Preocupação. Seis crianças salientaram preocupar-se com o bem-estar do irmão doente: "Preocupado, porque tem medo que aconteça uma coisa muito grave ao irmão dele (I., irmã com 7 anos de criança com neurofibromatose I com glioma das vias óticas)". A literatura corrobora este resultado, reforçando que a imprevisibilidade da doença e das suas consequências no futuro geram um aumento da ansiedade dos irmãos (Bayliss, 2007). Duas crianças referiram também preocupação associada ao agravamento da doença: "Às vezes pode ter medo porque ele pode ficar mal e pode ter medo que ele fique com uma doença grave (G., irmão com 9 anos de criança com síndrome genético ATR-x)".

Tristeza. Cinco irmãos disseram sentir-se tristes, sendo que três destes associaram a sua tristeza à doença (Prchal \& Landolt, 2012): "Sente-se triste porque o seu irmão está doente (F., irmão com 7 anos de criança com síndrome genético ATR-x)". A tristeza foi também referida como resultante da incompreensão da condição do irmão e da privação de convívio durante as hospitalizações ou a escola (Sargent et al., 1995).

Medo. O medo foi referido por três irmãos saudáveis, sobretudo quanto ao desconhecido e às consequências graves na saúde da criança doente, como referido pela literatura (Fleary \& Heffer, 2013).

Satisfação pelas melhorias de saúde. Três irmãos referiram também sentir-se satisfeitos quando observam melhorias de saúde no seu irmão doente (Gomes, Amador, \& Collet, 2012): "E quando o irmão ficou bom, ele ficou muito contente e ficou o irmão muito feliz. Eles os dois ficaram muito felizes e ele perguntou 'estou feliz por tu ires a casa' (S., irmã com 6 anos de criança com bulbomielite idiopática com insuficiência respiratória com dependência total de ventilação)".

Empatia. A empatia do irmão saudável pela criança doente é também frequente nos irmãos saudáveis, embora dependa da relação fraterna, da idade e de características individuais e contextuais (Nolbris, Enskär, \& Hellström, 2007). Neste estudo, a empatia foi manifestada em relação ao irmão doente - "Ele deve sentir-se mal por não poder brincar com as outras crianças, mas há muitas brincadeiras que ele pode (G., irmão com 8 anos de criança com trissomia 21 associada a cardiopatia congénita, dificuldades renais e respiratórias e hipotiroidismo)" -, mas também com a sua família e com pessoas socialmente desfavorecidas: "[No futuro] vão gostar muito uns dos outros. Vão ajudar muito as outras pessoas e vão dar comida aos pobres, e dinheiro (F., irmão com 7 anos de criança com síndrome genético ATR-x)". 
Para além das subcategorias acima descritas, destacam-se também o arrependimento, a irritabilidade (Wilkins, 2002), a manifestação de queixas somáticas (Sidhu, Passmore, \& Maker, 2005) e a brincadeira solitária. Apesar da literatura identificar o ciúme, o ressentimento, a raiva e a vergonha como emoções também frequentes nos irmãos saudáveis, esses resultados não foram encontrados no presente estudo.

\section{Ocupação de tempos livres e responsabilidades}

A literatura identifica uma privação de oportunidades de lazer para os irmãos saudáveis (Gomes et al., 2013), aspeto referido apenas por um dos participantes. De igual forma, Prchal e Landolt (2012) também referem um aumento de responsabilidades associadas à participação nas tarefas domésticas, o que foi referido também apenas por um dos irmãos: "A mãe vai fazer a massa e o Joãozinho vai ajudar (C., irmã com 6 anos de criança com paralisia cerebral profunda)". Três das crianças participantes referiram a sua participação no cuidado ao irmão doente, dizendo que ajudam a sua família e que levam o irmão doente ao colo, aspeto identificado na literatura como fundamentalmente positivo no seu processo de adaptação (Essen \& Enskär, 2003).

Como ocupação dos seus tempos livres, as crianças referiram alguns hobbies, como as idas à praia e ao parque, o poderem apreciar a natureza e as atividades manuais, como os desenhos e os recortes: "Vai à piscina, vai ao mar, vai à praia, vai brincar com os seus amigos ao parque e à escola (D., irmão com 6 anos de criança com paralisia cerebral profunda)". Para Essen e Enskär (2003), é fundamental que se proporcionem oportunidades prazerosas e de diversão para os irmãos. Para além disso, duas crianças destacaram também as atividades em família, como os lanches no parque e os jogos de cartas, recurso adaptativo importante segundo a literatura (Barros, 2010).

Relação com os pares. Três dos irmãos enfatizaram os momentos de brincadeira com os pares e, num dos casos, o recurso a esta interação como uma estratégia de distração: "Mas se calhar continuou um bocado triste, mas depois foi brincar com os amigos e já não se lembrou. Depois esqueceu-se do irmão (S., irmã com 6 anos de criança com bulbomielite idiopática com insuficiência respiratória com dependência total de ventilação)". Estes resultados são corroborados pelo estudo de Oros (2017), o qual concluiu que as crianças do sexo feminino desenvolvem crenças de autoeficácia mais sólidas no âmbito social e académico, sendo reforçadas no seu comportamento de cooperação e de troca emocional. Por sua vez, também o estudo de Alderfer e Hodges (2010) concluiu que o apoio dos amigos é considerado o mais importante e frequente para os irmãos saudáveis, contribuindo também para a redução de sintomatologia associada a ansiedade e depressão.

Escola. Murray (2000) destaca o investimento e o recurso às relações extrafamiliares como tendo um papel moderador no impacto da doença na adaptação destes irmãos, contribuindo assim para o seu bem-estar psicológico. A maioria dos irmãos incluíram a escola no seu discurso, referindo as dinâmicas e rotinas escolares. Contudo, uma criança destacou o recurso aos professores para apoio emocional como um recurso protetor: "Aqui está com cara um bocado triste. Porque... se calhar foi pelo irmão. $O$ irmão disse-lhe 'Eu ainda estou doente, mas se calhar posso ir lá a casa visitar-te ou na escola'. Mas ele ficou na mesma triste, então a professora tentou escrever e ele disse-lhe tudo: 'Estou triste por causa do meu irmão ficar lá ainda no hospital' (S., irmã com 6 anos de criança com bulbomielite idiopática com insuficiência respiratória com dependência total de ventilação)".

Rotinas familiares em casa. Todos os participantes fizeram alusão às suas rotinas durante a permanência em casa, destacando o descanso, a brincadeira e as refeições como as principais dinâmicas (Patterson et al., 2011): "Podem ir tomar banho e ir jantar. Depois da escola... ou talvez os irmãos podem brincar e a mãe ir fazer o jantar. E depois o pai chega e vão jantar (G., irmão com 9 anos de criança com síndrome genético ATR-x)".

\section{Perspetivas sobre o futuro}

Para Essen e Enskär (2003), os irmãos saudáveis tendem a manifestar necessidade de falar sobre a doença e o futuro. No presente estudo, todas as crianças abordaram esse assunto, embora três delas tenham demonstrado alguma hesitação em fazê-lo, manifestando silêncios 
prolongados. Este resultado é corroborado pelo estudo de Sidhu e colegas (2005) que concluiu que a perda de controlo das rotinas e planos expectáveis para o futuro é causador de preocupações e sintomatologia de ansiedade. Por outro lado, este resultado também pode ser explicado pelo medo associado à imprevisibilidade da doença do irmão e das suas implicações (Fleary e Heffer, 2013).

Positividade. Quatro crianças apelaram à positividade no seu discurso sobre o futuro: "Acho que ele deve ficar muito contente porque tem o vosso pai, a mãe e o vosso irmão. Então eles iam ficar felizes e iam pensar sempre com o vosso irmão (S., irmã com 6 anos de criança com bulbomielite idiopática com insuficiência respiratória com dependência total de ventilação)". Helgeson e Cohenh (1996) sugerem a ressignificação da experiência de uma forma positiva como uma estratégia de adaptação eficaz, através da promoção do aumento do controlo percebido e do otimismo sobre o futuro.

Tarefas expectáveis no ciclo vital da família. Quatro irmãos apelaram a tarefas consideradas normativas no ciclo vital do sistema familiar, como a construção de uma família, o casamento, a mudança de casa, a dedicação ao estudo para futuramente possibilitar a escolha de um curso, o emprego e o ser idoso (Carter \& McGoldrick, 2005): "Vão sempre crescendo mais... vai haver uma certa altura que eles vão arranjar e vão ir para outra casa. Vão ter de tirar o seu próprio curso, estudar muito, provavelmente para os exames do 8. ${ }^{\circ}$... 9..$^{\circ}$, é o 9. ${ }^{\circ}$ ano e do $12 .^{\circ}$ (J., irmão com 9 anos de criança com fibrose quística)".

Foco no irmão doente. Dois dos irmãos saudáveis referiram-se ao futuro como mantendo o foco no irmão doente, nomeadamente através do apoio constante e da preocupação com a sua doença (Fleary \& Heffer, 2013): “Acho que... que iam ficar preocupados. Que iam ajudar muito o irmão do Joãozinho... e... e é só... (I., irmã com 7 anos de criança com neurofibromatose I com glioma das vias óticas)".

\section{Relação fraterna}

A relação entre os irmãos foi referida num total de 48 vezes por todos os participantes.
Apresentam-se as subcategorias mais abordadas pelas crianças participantes deste estudo.

Brincadeira com o irmão doente. Seis dos irmãos saudáveis referiram brincar com o seu irmão doente, embora esta tenha de ser frequentemente controlada e com a supervisão dos adultos cuidadores (Fernandes, 2005): "Depois fomos lá fora, brincámos, vimos flores, duas flores, o sol e ficámos felizes. Então brincámos e corremos... (S., irmã com 6 anos de criança com bulbomielite idiopática com insuficiência respiratória com dependência total de ventilação)".

Foco no irmão doente. Quatro dos irmãos saudáveis destacaram a vontade de agradar o irmão doente (Essen \& Enskär, 2003): "Iam dar um passeio pelo um passeio que viam uma paisagem linda! Para o irmão ficar alegre (P., irmão com 9 anos de criança com paralisia cerebral profunda)". Por outro lado, duas das crianças manifestaram sentir orgulho no irmão, sendo colocada a hipótese de os vínculos fraternos se fortalecerem com a experiência de doença (Nolbris et al., 2007).

Afetividade. Três irmãos referiram os afetos que sentiam pelo seu irmão doente: "Era uma vez uma menina que se chamava I. [nome da própria criança], que tinha um irmão que se chamava $M$. [nome do irmão doente]. E ela gostava muito do irmão dela. Um dia foram os dois brincar lá para fora e... e estava sol. E eles pediram para ir brincar para a piscina (I., irmã com 7 anos de criança com neurofibromatose I com glioma das vias óticas)". Segundo Mendonça (2000), uma relação fraterna caracterizada por ser calorosa contribui para uma melhor adaptação à doença do irmão.

Interação entre irmãos saudáveis. A literatura refere que o tamanho da fratria superior a dois irmãos - a criança doente e dois ou mais irmãos saudáveis - é protetora de perturbações psicológicas (Mendonça, 2000). Uma vez que este estudo também integrou fratrias com mais de um irmão saudável, surgiram dados relativos à relação estabelecida entre os mesmos. Quatro crianças referiram a brincadeira com o seu irmão saudável: "Aqui, a mana está aqui, a ir pedir-me para nós a irmos meter no baloiço. E o mano [doente] está na espreguiçadeira e nós estamos a jogar futebol. E eu aqui de joelhos, sou eu a defender a bola! 
(G., irmão com 9 anos de criança com síndrome genético ATR-x)". Uma criança manifestou reconhecer a preocupação que o seu irmão saudável também sentia em relação ao irmão doente.

Importa referir que não foram mencionados conflitos ou hostilidade na relação fraterna (Barbarin et al., 1995). Por outro lado, os dados deste estudo não foram analisados à luz do tamanho da fratria, da diferença de idades ou sexo dos irmãos saudáveis, devido ao seu carácter descritivo-exploratório. Também decorrente desta natureza, emergiram resultados no âmbito das dinâmicas familiares e da fratria imaginária, inicialmente não contemplados nos objetivos específicos desta investigação. Estas duas categorias principais resultaram da análise dos dados obtidos através das entrevistas semiestruturadas e das narrativas do desenho sobre a fratria.

\section{Dinâmicas familiares}

A preocupação constante com a criança doente e o seu bem-estar altera, com frequência, as dinâmicas do sistema familiar (Brown, Coad, \& Franklin, 2017), como referido por todos os participantes.

Interação com a família nuclear. Quase todas as crianças referiram a proximidade que sentiam com a sua mãe. Em relação ao seu pai, quatro das crianças referem a proximidade afetiva que sentem com o mesmo e duas evidenciam a perceção que têm da falta de convívio com o mesmo: "Os pais [vão fazer o lanche]! Provavelmente é só a mãe, o papá nunca está em casa (J., irmão com 9 anos de criança com fibrose quística)". Este resultado pode ser explicado pelo facto de as mães assumirem, com frequência, o papel de cuidadoras principais dos filhos doentes e por isso estarem mais presentes durante o tempo que os irmãos saudáveis estão em casa. As características da amostra deste estudo evidenciam também este aspeto, uma vez que todos os cuidadores são mulheres, maioritariamente as mães. Os pais, por assumirem frequentemente as responsabilidades de natureza financeira e de sustento da sua família, partilham menos tempo com os seus filhos (Mesman et al., 2013).
Coesão familiar. A maioria dos participantes apelaram à coesão da sua família, sobretudo através da narrativa que construíram do desenho sobre a sua fratria, como evidenciado na seguinte citação: "A piscina era muito funda e o $M$. [nome do irmão doente] não conseguia tocar com os pés no chão, por isso precisava de uma boia. E a I. [nome da própria criança] nadava sozinha. $E$ davam mergulhos... e depois sairam da piscina e ainda brincaram às cartas com a mãe deles (I., irmã com 7 anos de criança com neurofibromatose I com glioma das vias óticas)". Este aspeto é também encontrado no estudo de Dockerty, Skegg e Williams (2003), que concluiu que a doença crónica complexa da criança ou jovem pode constituir um fator de coesão familiar.

Apoio da família alargada. Três das crianças mencionaram o apoio e a participação de membros da família alargada nas suas rotinas, nomeadamente a avó e os primos: "Às vezes, eu, o mano [saudável], a mana e a avó. Ou outras vezes, eu, o mano, a mana e a mãe (F., irmão com 7 anos de criança com síndrome genético ATRx)". O estudo de Dilworth-Anderson (2008) apoia este resultado, afirmando que os avós desempenham um papel importante de apoio aos cuidadores principais, assim como às próprias crianças doentes e aos seus irmãos saudáveis. $\mathrm{O}$ apoio dos avós pretende muitas vezes colmatar a lacuna ao apoio dos pais, os quais se encontram a maior parte do dia fora de casa, a trabalhar.

Comunicação na família. Os pais referem um esforço ativo na promoção de uma comunicação aberta e honesta na família (Sidhu et al., 2005), o que constitui uma estratégia de coping eficaz (Björk et al., 2005). Neste estudo, apenas uma criança fez referência a esta comunicação, salientando o questionamento que existe por parte das crianças saudáveis aos seus pais, as explicações por parte dos adultos cuidadores e a participação ativa de todos os membros (Eapen, Mabrouk, \& Bin-Othman, 2008). Neste seguimento, é de particular relevância assumir que não deve haver negligência em relação ao apoio e orientações dadas aos pais. Espada, Grau e Fortes (2010) destacam a necessidade de transmitir e ensinar estratégias de coping, permitindo assim um encorajamento ao nível da comunicação e uma maior expressão de sentimentos e preocupações. 
Para além das subcategorias anteriores, também foi referida a afetividade sentida na família, a sua alegria e o orgulho na mesma: "Ele [o Joãozinho] vai surpreender o irmão com a levá-lo a passear ou a lhe dar alguma coisa para ele brincar, um boneco. E à família, pode dar um diploma a dizer que cuida muito bem dele, do irmão (P., irmão com 9 anos de criança com paralisia cerebral profunda)".

\section{Fratria imaginária}

É de salientar que, numa das fratrias com mais de dois elementos, uma das crianças refere-se ao seu irmão saudável como estando doente, dizendo: "Este é o P. [nome do irmão saudável] com a cabeça laranja desta vez. Agora está com mais febre. Porque aqui tinha a cabeça amarela e aqui tem a cabeça laranja... deve estar com muita febre! (...) Vou fazê-lo muito feio, muito feio mesmo! A boca preta... preta, muito preta! (...) Ficou com a boca queimada (C., irmã com 6 anos de criança com paralisia cerebral profunda)". Este resultado pode ser discutido atendendo a que, por vezes, os irmãos saudáveis manifestam o sentimento de culpa por eles próprios serem saudáveis (Fernandes, 2005), e neste caso em particular, projetando-se no outro irmão saudável. Por outro lado, reflete também a confusão associada à vivência psicológica da doença do irmão, refletindo os seus medos, imaturidade e fantasias (Cheron \& Pettengill, 2011).

Três crianças saudáveis referiram também que os seus irmãos doentes estão saudáveis. Destacam a ausência de vigilância constante, a comunicação verbal e a autonomia na mobilidade: "Só uma pergunta: posso desenhar que o meu irmão fala? (P., irmão com 9 anos de criança com paralisia cerebral profunda)" e "Esta sou eu e estava eu e o $G$. [nome do irmão doente] e estávamos a brincar lá fora, no jardim. Estava sol, estávamos a jogar à apanhadinha. E era ele a caçar-me e depois eu cacei-o a ele e depois encontrámos uma árvore com um pássaro (S., irmã com 6 anos de criança com bulbomielite idiopática com insuficiência respiratória com dependência total de ventilação)". Estes resultados são congruentes com as vivências emocionais descritas anteriormente, evidenciando a tristeza pela condição de saúde do irmão doente e as suas incapacidades, assim como pela impossibilidade de partilharem diálogos e atividades habituais numa fratria onde todos os membros sejam saudáveis (Prchal \& Landolt, 2012). É assim transmitido um desejo profundo de que a doença não fizesse parte das suas vidas e não condicionasse a dinâmica da fratria.

Importa, contudo, apresentar as limitações do presente estudo, como não ter atingido a saturação teórica, devido ao número reduzido de participantes e ao grau de dificuldade de acesso a esta população. Por outro lado, e no âmbito da idade escolar, os participantes tinham entre 6 e 9 anos, o que não permitiu explorar a experiência psicológica de irmãos com idade compreendida entre os 10 e os 12 anos. A diversidade de doenças crónicas complexas que foram incluídas na amostra e a heterogeneidade de fratrias constituem também limitações deste estudo.

Por ser um estudo descritivo-exploratório, pretende abrir caminho para novas linhas de investigação, como explorar a vivência da rivalidade fraterna, aprofundar o conhecimento sobre os fatores protetores na adaptação à doença e compreender a experiência dos irmãos saudáveis durante as hospitalizações da criança ou jovem doente. Para além disso, sugerem-se estudos com amostras de maior dimensão, que se foquem noutras faixas etárias e que se fundamentem numa linha de positividade e, por isso, estudem dimensões como a esperança e o sentido de humor.

Recomenda-se também a inclusão de variáveis descritas na literatura como mediadoras do processo de adaptação dos irmãos, tais como o género, a posição na fratria, a dimensão da família e as especificidades de cada doença. Por último, sugerem-se estudos que aprofundem a experiência psicológica de outros membros da família, como os avós.

\section{Considerações Finais}

O presente estudo contribui para a identificação das especificidades da experiência psicológica de uma doença crónica complexa na fratria em idade escolar. Este conhecimento reforça a necessidade e pertinência do desenvolvimento de intervenções específicas junto desta população, promovendo a sua capacidade de 
resiliência, a adaptação psicológica a esta experiência de adversidade e o seu crescimento pós-traumático. É igualmente importante a sensibilização dos adultos cuidadores, sobretudo os pais, através de intervenções psicoeducacionais, para o apoio informativo e emocional necessários aos filhos saudáveis. A promoção de competências e estratégias de coping em todos os elementos da família é fundamental, uma vez que o objetivo último é também a promoção da harmonia e resiliência familiares.

\section{Referências}

Alderfer, M. A., \& Hodges, J. A. (2010). Supporting siblings of children with cancer: A need for family-school partnerships. School Mental Health, 2(2), 72-81.

Alderfer, M. A., Long, K. A., Lown, E. A., Marsland, A. L., Ostrowski, N. L., Hock, J. M., \& Ewing, L. J. (2010). Psychosocial adjustment of siblings of children with cancer: A systematic review. Psychooncology, 19(8), 789-805.

Association for Children with Life-Threatening or Terminal Conditions and their Families and the Royal College of Paediatrics and Child Health (ACT \& RCPCH). (2009). A guide to the development of children's palliative care services $\left(3^{\text {rd }}\right.$ ed.). Bristol, United Kingdom.

Azeredo, Z., Amado, J., Silva, H. N. A., Marques, I. G., \& Mendes, M. G. V. (2004). A família da criança oncológica: Testemunhos. Acta Médica Portuguesa, 17(1), 375-380.

Barbarin, O., Sahler, O., Carpenter, P., Copeland, D., Dolgin, R., Roghmann, K., et al. (1995). Sibling adaptation to childhood cancer collaborative study: Parental views of pre- and postdiagnosis adjustment of siblings of children with cancer. Journal of Psychosocial Oncology, 13(3), 1-20.

Barros, L. (2003). Psicologia pediátrica: Uma perspectiva desenvolvimentista. (2. ${ }^{\mathrm{a}}$ ed.). Lisboa: Climepsi Editores.

Barros, L. (2010). Família, saúde e doença: Intervenções dirigidas aos pais. Alicerces, III(3), 207-221.

Bayliss, J. (2007). Commentary on experience of siblings of children treated for cancer.
European Journal of Oncology Nursing, 11, 115-116.

Bernardes, I. A., \& Pegoraro, R. F. (2015). Irmãos saudáveis de criança com câncer: Revisão integrativa da literatura. Revista Espaço para a Saúde (Londrina), 16(4), 98-108.

Besier, T., Hölling, H., Schlack, R., West, C., \& Goldbeck, L. (2010). Impact of a familyoriented rehabilitation programme on behavioral and emotional problems in healthy siblings of chronically ill children. Child: care, health and development, 36(5), 686-695.

Björk M., Wiebe T., \& Hallström I. (2005). Striving to survive: families' lived experiences when a child is diagnosed with cancer. Journal of Pediatric Oncology Nursing, 22(5), 265-275.

Braun, V., \& Clarke, V. (2006). Using thematic analysis in psychology. Qualitative Research in Psychology, 3(2), 77-101.

Brown, E., Coad, J., \& Franklin, A. (2017). The impact of a sibling's life-limiting genetic condition on adult brothers and sisters. American Journal of Medical Genetics Part A, 173(7), 1754-1762.

Cardoso, F. T. (2007). Câncer infantil: Aspectos emocionais e atuação do psicólogo. Revista da Sociedade Brasileira de Psicologia Hospitalar, 10(1), 25-52.

Carr, A. (2006). The handbook of child and adolescent clinical psychology: A contextual approach. $2^{\text {nd }}$ Edition. Hove: Routledge.

Carter, E., \& McGoldrick, M. (2005). Overview: The expanded life cycle: Individual, family, and social perspectives. In E. Carter \& M. McGoldrick (Eds.), The expanded life cycle: Individual, family, and social perspectives (pp. 1-26). Boston: Allyn \& Bacon.

Cheron, M., \& Pettengill, M. (2011). Experiência do irmão sadio em relação à doença e hospitalização do irmão com câncer. Acta Paulista de Enfermagem, 24(5), 605-610.

Dilworth-Anderson, P. (2008). The importance of grandparents in extended-kin caregiving to black children with sickle cell disease. Journal of Health \& Social Policy, 5(3-4), 185-202.

Dockerty, J. D., Skegg, D. C. G., \& Williams, S. M. (2003). Economic effects of childhood 
cancer on families. Journal of Paediatric Child Health, 39(4), 254-258.

Eapen, V., Mabrouk, A., \& Bin-Othman, S. (2008). Attitudes, perceptions, and family coping in pediatric cancer and childhood diabetes. Annals of the New York Academy of Sciences, 1138, 47-49.

Espada, M. C., Grau, C., \& Fortes, M.C. (2010). Teaching coping strategies to parents of children suffering from cancer using a short film. Anales del sistema sanitario de Navarra, 33(3), 259-269.

Essen, V. L., \& Enskär, K. (2003). Important aspects of care and assistance for siblings of children treated for cancer: A parent and nurse perspective. Cancer Nursing, 26(3), 203-210.

Fernandes, O. M. (2005). Ser único ou ser irmão: As relações entre os irmãos nas famílias actuais. Lisboa: Oficina do Livro.

Feudtner, C., DiGiuseppe, D. L., \& Neff, J. M. (2003). Hospital care for children and young adults/in the last year of life: A populationbased study. BMC Medicine, 1(3), 1-9.

Fleary, S. A., \& Heffer, R. W. (2013). Impact of growing up with a chronically ill sibling on well siblings' late adolescent functioning. ISRN Family Medicine, 2013, 1-8.

Goldman, A., Hain, R., \& Liben, S. (2012). Oxford textbook of palliative care for children $\left(2^{\text {nd }}\right)$. London and New York: Oxford University Press.

Gomes, I. P., Amador, D. D., \& Collet, N. (2012). A presença de familiares na sala de quimioterapia pediátrica. Revista Brasileira de Enfermagem, 65(5), 803-808.

Gomes, I. P., Lima, K. A., Rodrigues, L. V., Lima, R. A., \& Collet, N. (2013). Do diagnóstico à sobrevivência do câncer infantil: Perspectiva das crianças. Texto Contexto Enfermagem, 22(3), 671-679.

Hartling, L., Milne, A., Tjosvold, L., Wrightson, D., Gallivan, J., \& Newton, A. S. (2014). A systematic review of interventions to support siblings of children with chronic illness or disability. Journal of Paediatrics and Child Health, 50(10), 26-38.

Helgeson, V. S., \& Cohen, S. (1996). Social support and adjustment to cancer: Reconciling descriptive, correlational, and intervention research. Health Psychology, 15, 135-148.
Jurbergs, N., Long, A., Ticona, L., \& Phipps, S. (2009). Symptoms of posttraumatic stress in parents of children with cancer: Are they elevated relative to parents of healthy children?. Journal of Pediatric Psychology, 34(1), 4-13.

Lobato, D. J., \& Kao, B. T. (2002). Integrated sibling-parent group intervention to improve sibling knowledge and adjustment to chronic illness and disability. Journal of Pediatric Psychology, 27, 711-716.

McDonald, F. E. J., Patterson, P., White, K. J., Butow, P., \& Bell, M. L. (2014). Predictors of unmet needs and psychological distress in adolescent and young adult siblings of people diagnosed with cancer. Psycho-Oncology, 24(3), 333-340.

Mendonça, M. (2000). A adaptação psicológica em irmãos de crianças e jovens com paralisia cerebral (Dissertação de Mestrado não publicada). Instituto de Educação e Psicologia da Universidade do Minho, Braga.

Mesman, G. R., Kuo, D. Z., Carroll, J. L., \& Ward, W. I. (2013). The impact of technology dependence on children and their families. Journal of Pediatric Health Care, 27(6), 451459.

Miceli, A., \& Zornig, S. (2012). Câncer infantojuvenil: $\mathrm{O}$ trauma dos irmãos. Tempo psicanalítico, 44(1), 11-26.

Murray, J. S. (2000). A concept analysis of social support as experienced by siblings of children with cancer. Journal of Pediatric Nursing, 15(5), 313-322.

Murray, J. S. (2002). A qualitative exploration of psychosocial support for siblings of children with cancer. Journal of Pediatric Nursing, 17, 327-337.

Navarausckas, H. B., Sampaio, I. B., Urbini, M. P., \& Costa, R. C. V. (2010). Ei, eu também estou aqui: Aspectos psicológicos da percepção de irmãos frente à presença de uma criança com paralisia cerebral no núcleo familiar. Estudos de Psicologia (Campinas), 27(4), 505-513.

Nogueira, A. J., \& Francisco, R. (2017). Autoavaliação da qualidade de vida familiar em cuidados paliativos pediátricos:Um estudo exploratório. Revista Cuidados Paliativos, 4(1), 6-15. 
Nolbris M., Enskär K., \& Hellström A.L. (2007). Experience of siblings of children treated for cancer. European Journal of Oncology Nursing, 11(2), 106-116.

Opperman, S., \& Alant, E. (2003). The coping responses of the adolescent siblings of children with severe disabilities. Disability and Rehabilitation, 25, 441-454.

Oros, L. B. (2017). Valores normativos de la Escala Multidimensional de Autoeficacia Infantil para población argentina. Revista Iberoamericana de Diagnóstico y Evaluación - e Avaliação Psicológica, 2(44), 172-181.

Pai, A. L. H., Drotar, D., Zebracki, K., Moore, M., \& Youngstrom, E. (2006). A meta-analysis of the effects of psychological interventions in pediatric oncology on outcomes of psychological distress and adjustment. Journal of Pediatric Psychology 31(9), 978988.

Patterson P., Millar B., \& Visser, A. (2011). The development of an instrument to assess the unmet needs of young people who have a sibling with cancer: Piloting the Sibling Cancer Needs Instrument (SCNI). Journal of Pediatric Oncology Nursing, 28(1), 16-26.

Pelchat, D., Ricard, N., Bouchard, J. M., Perreault, M., Saucier, J. F., Berthiaume, M., \& Bisson, J. (2001). Adaptation of parents in relation to their 6-month. old infant's type of disability. Child Care, Health and Development, 25(5), 377-398.

Prchal, A., \& Landolt M. A. (2009). Psychological interventions with siblings of pediatric cancer patients: A systematic review. Psychooncology, 18(12), 1241-1251.

Prchal A., \& Landolt, M. A. (2012). How siblings of pediatric cancer patients experience the first time after diagnosis: A qualitative study. Cancer Nursing, 35(2), 133-140.

Rocha, C. (2015). Adaptación Psicológica de los hermanos de niños con câncer (Tesis Doctoral no publicada). Universidad Miguel Hernández de Elche, Sant Joan d'Alacant, Espanha.

Rodriguez, E. M., Dunn, M. J., Zuckerman, T., Vannatta, K., Gerhardt, C. A., \& Compas B. E. (2012). Cancer-related sources of stress for children with cancer and their parents. Journal of Pediatric Psychology, 37(2), 185197.
Román, M., Hodges, J., Palacios, J., Moreno, C., \& Hillman, S. (2018). Evaluación de las representaciones mentales de apego a través de las historias incompletas: Aplicación española de Story Stem Assessment Profile (SSAP). Revista Iberoamericana de Diagnóstico y Evaluación - e Avaliação Psicológica, 1(46), 5-19.

Sargent, J. R., Sahler, O. J. Z., Roghmann, K. J., Mulhern, R. K., Barbarian, O. A., Carpenter, P. J., Copeland, P. J., Dolgin, M. J., \& Zeltzer, L. K. (1995). Sibling adaptation to childhood cancer collaborative study: Sibling's perceptions of the cancer experience. Journal of Pediatric Psychology, 20, 151-164.

Seligman, M. (1983). Sources of psychological disturbance among siblings of handicapped children. Journal of Counseling \& Development, 61(9), 529-531.

Sidhu, R., Passmore, A., \& Baker, D. (2005). An investigation into parent perceptions of the needs of siblings of children with cancer. Journal of Pediatric Oncology Nursing, 22(5), 276-287.

Svavarsdottir, E. K., \& Sigurdardottir, A. O. (2006). Developing a family-level intervention for families of children with cancer. Oncology Nursing Forum, 33, 983990.

Vermaes, I. P. R., van Susante, A. M. J., \& Bakel, H. J. A. (2012). Psychological functioning of siblings in families of children with chronic health conditions: A meta-analysis. Journal of Pediatric Psychology, 37(2), 166-184.

White, M. (2005). Children, trauma and subordinate storyline development. International Journal of Narrative Therapy \& Community Work, 2005(3/4), 10-22.

Wilkins, K. (2002). Sibling adaptation to the family crisis of childhood cancer. Cancer Oncology Nursing Journal, 13, 46-52.

Woodgate, R. L. (2006). Siblings' experiences with childhood cancer. Cancer Nursing, 29, 406-414.

World Health Organization (WHO) (1998). Cancer pain relief and palliative care in children. Geneva: WHO. Disponível em: http://whqlibdoc.who.int/publications/924154 5127.pdf. 